$$
F(\mathrm{i} \beta)=\int_{-\infty}^{\infty} \frac{v^{2} \varphi \mathrm{d} v}{v^{2}+\beta^{2}} .
$$

If $k^{2}$ is rewritten as

$$
k^{2}=\nu^{2} / \beta^{2},
$$

where $\nu$ is the growth rate $\omega=i \nu$, then (3) appears as

$$
\nu^{2} / \beta^{2}=\int_{-\infty}^{\infty} \frac{v^{2} \varphi \mathrm{d} v}{v^{2}+\beta^{2}} .
$$

Both sides of (9) are plotted in fig. 1.

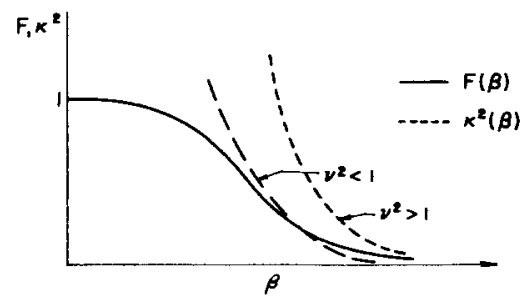

Fig. 1.

The inequalities which are depicted in fig. 1 are obtained by examining the asymptotic behaviour of $F$ for large $\beta$, according to which

$$
F \sim \frac{1}{\beta^{2}}\left(1-\frac{3}{\beta^{2}}+\ldots\right) .
$$

It follows that for large $\beta$ both $k^{2}$ and the first derivative of $k^{2}$ are larger than the related values of $F$ if $\nu^{2}>1$, which, in dimensional form, appears as

$$
v>\omega_{0} \text {, }
$$

whence there are no solutions for $\nu>\omega_{0}$ and $\omega_{0}$ represents the maximum growth rate of these instabilities.

As a concluding remark we note that all of the remaining modes are damped. The exact forms are obtained by continuing $F$ analytically into the lower half $z$-plane. The only physically relevant fact about these damped modes is that some of them propagate (viz., the roots that lie off the imaginary axis).

\section{References}

1) J.Jeans, Phil. Trans. A 199 (1902) 49.

2) D. Lynden-Bell, Monthly Notices Roy. Astron. Soc. 124 (1962) 279.

3) R.Simon, Bull. Acad. Roy. Belg. series 5, 47 (1962) 7.

4) R. L. Liboff, Gravitational instability and one-component plasma oscillations (New York University, NYO9754, November 1962).

5) P.Debye and E. Huckel, Phys. Z. 24 (1923) 185.

\title{
REGGE POLE HYPOTHESIS AND POLARISATIONS IN $\pi p$ AND Kp SCATTERING
}

\section{H. ÜBERALL *}

Harrison M. Randall Laboratory of Physics, University of Michigan, Ann Arbor, Michigan

Received 2 January 1963

Regge pole hypothesis 1 ) permits one to obtain the asymptotic behaviour of differential and total cross sections in the s channel for high energy, by assuming particles corresponding to the trajectories in the Chew-Frautschi diagram 2 ) being exchanged in the $t$ channel of the same reaction. The high energy behaviour of various total cross sections was obtained in this way by Udgaonkar 3). Gribov and Pomeranchuk ${ }^{4)}$ have pointed out that polarisations depend on interferences between various exchanged trajectories, thus providing additional information on the trajectories and their couplings. Polarisations were obtained by Hara 5) for NN scattering. In this paper, expressions for the polarisations are presented for $\pi \mathrm{N}$ and $\mathrm{KN}, \overline{\mathrm{K}} \mathrm{N}$ scattering, together with differential and total cross sections.

Using the general form of the scattering matrix for a zero-spin and a Dirac particle 6), we obtair for the differential cross section, asymptotically for $s \rightarrow \infty$

$$
\frac{\mathrm{d} \sigma}{\mathrm{d} t} \approx \frac{1}{4 \pi \delta}\left(\frac{m^{2}}{s}\left|A+\frac{s}{2 m} B\right|^{2}-\frac{t}{4 s}|A|^{2}\right),
$$

\footnotetext{
* Supported in part by the Office of Naval Research, U.S. Navy.
} 
and likewise for the polarisation

$$
P \approx \frac{1}{2} \sqrt{-t} \operatorname{Im} B^{*} A\left(\frac{m^{2}}{s}\left|A+\frac{s}{2 m} B\right|^{2}-\frac{t}{4 s}|A|^{2}\right) ;
$$

the optical theorem further gives

$$
\sigma^{\text {tot }} \approx \operatorname{Im}\left(\frac{2 m}{s} A+B\right)_{t=0} .
$$

Here, $m$ is the mass of the Dirac particle, $s$ the squared total c.m. energy in the s channel, and $t$ is the squared momentum transfer, related to the scattering angle by

$$
\cos \theta \approx 1+(2 t / s) \text {. }
$$

The polarisation is given along the direction $\boldsymbol{k}^{\prime} \times \boldsymbol{k}$, where $\boldsymbol{k}$ is the momentum of the incoming and $\boldsymbol{k}^{\prime}$ that of the outgoing boson. The equations are correct if terms of order $\mathrm{m}^{2} / \mathrm{s}$ are negligible; $|t|$ has been assumed to reach up to order $m^{2}$.

1. $\pi p$ scattering. Here, the polarisation is already given implicitly in ref. 1 , the cross sections explicitly. For elastic scattering, $\pi^{ \pm}+\mathrm{p} \rightarrow \pi^{ \pm}+\mathrm{p}$, one has to replace

$$
A \rightarrow A^{+} \mp A^{-}, \quad B-B^{+} \mp B^{-},
$$

for charge exchange scattering, $\pi^{-}+\mathrm{p} \rightarrow \pi^{\mathrm{O}}+\mathrm{n}$,

$$
A \rightarrow-\sqrt{2} A^{-}, \quad B \rightarrow-\sqrt{2} B^{-} \text {. }
$$

From isotopic spin and $G$ parity considerations, only the $P, P^{\prime}$ and $A B C$ trajectories $(T=0, G=+)$ can contribute to $A^{+}, B^{+}$and only $\rho(T=1, G=+)$ to $A^{-}, B^{-}$. One has

$$
\begin{aligned}
& A^{+}=-\frac{s_{\mathrm{O}}}{2 m}\left\{\frac{1+\mathrm{e}^{-\mathrm{i} \pi \alpha_{P}}}{\sin \pi \alpha_{P}}\left(\frac{s}{s_{\mathrm{O}}}\right)^{\alpha_{P}} b_{A P^{+}}+\frac{1+\mathrm{e}^{-\mathrm{i} \pi \alpha_{P^{\prime}}}}{\sin \pi \alpha_{P^{\prime}}}\left(\frac{s}{s_{\mathrm{o}}}\right)^{\alpha_{P^{\prime}}} b_{A P^{\prime}}+\frac{1+\mathrm{e}^{-\mathrm{i} \pi \alpha_{A B C}}}{\sin \pi \alpha_{A B C}}\left(\frac{s}{s_{\mathrm{O}}}\right)^{\alpha_{A B C}} b_{A, A B C}\right\}, \\
& B^{+}=-\frac{1+\mathrm{e}^{-\mathbf{i} \pi \alpha_{P}}}{\sin \pi \alpha_{P}}\left(\frac{s}{s_{\mathrm{O}}}\right)^{\alpha_{P^{-1}}} b_{B P}-\frac{1+\mathrm{e}^{-\mathrm{i} \pi \alpha_{P^{\prime}}}}{\sin \pi \alpha_{P^{\prime}}}\left(\frac{s}{s_{\mathrm{O}}}\right)^{\alpha P^{\gamma^{-1}}} b_{B P^{\prime}}-\frac{1+\mathrm{e}^{-\mathrm{i} \pi \alpha_{A B C}}}{\sin \pi \alpha_{A B C}}\left(\frac{s}{s_{\mathrm{O}}}\right)^{\alpha_{A B C^{-1}}} b_{B, A B C}, \\
& A^{-}=\frac{s_{\mathrm{o}}}{2 m} \frac{1-\mathrm{e}^{-\mathrm{i} \pi \alpha_{\mathrm{o}}}}{\sin \pi \alpha_{\rho}}\left(\frac{s}{s_{\mathrm{O}}}\right)^{\alpha_{\rho}} b_{A \rho}, \\
& B^{-}=\frac{1-\mathrm{e}^{-\mathbf{i} \pi \alpha_{\rho}}}{\sin \pi \alpha_{\rho}}\left(\frac{s}{s_{\rho}}\right)^{\alpha_{\rho}-1} b_{B \rho}
\end{aligned}
$$

The $\alpha_{P}(t), \alpha_{P_{r}}(t)$ etc. are the Regge trajectories, and the $b(t)$ are unknown residue functions of $t$. All are real analytic functions 7) with only right-hand cuts, thus are real for the physical values of $t<0$. The quantity $s_{\mathrm{O}}$ is an arbitrary parameter of dimension (mass) ${ }^{2}$, usually taken as $2 m^{2}$, or as $2 m \mu$, with $\mu$ the boson mass.

The polarisations then depend only on interferences of different trajectories and behave as *

$$
P \sim S^{\alpha} P^{i-\alpha} P \quad \text { or } \quad \sim S^{\alpha} c^{-\alpha_{P}},
$$

if the $P^{\prime}$ dominates the $\rho$ trajectory, or vice versa; in the former case, $P_{\pi^{+}}$and $P_{\pi^{-}}$have the same sign, in the latter, opposite sign. Charge exchange scattering has $P=0$, as it depends on one trajectory only. The total cross section is, with $b_{A i}+b_{B i}=\sigma_{i}(t)$

$$
\sigma_{\pi^{t} \mathrm{p}}^{\text {tot }}=\sigma_{P^{(}}(0)+\sigma_{P^{i}}(0)\left(\frac{s}{s_{\mathrm{O}}}\right)^{\alpha_{P^{\prime}}(0)-1}+\sigma_{A B C}(0)\left(\frac{s}{s_{\mathrm{O}}}\right)^{\alpha_{A B C}(0)-1} \mp \sigma_{\rho}(0)\left(\frac{s}{s_{\mathrm{O}}}\right)^{\alpha_{\rho}(0)-1} ;
$$

* Calling $b_{A i}+b_{B i}=\sigma_{i}(t), x_{i j}=\left[\epsilon_{i} \sin \pi a_{i}-\epsilon_{j} \sin \pi \alpha_{j}+\varepsilon_{i} \epsilon_{j} \sin \pi\left(\alpha_{i}-\alpha_{j}\right)\right]\left(\sin \pi \alpha_{i} \sin \pi \alpha_{j}\right)^{-1}$, where $\varepsilon_{i}$ is the signature of the $i$-th trajectory, and

$$
x_{i j}=x_{i j} \sigma_{i} \sigma_{j}\left(\frac{b_{A j}}{\sigma_{j}}-\frac{b_{A i}}{\sigma_{i}}\right)\left(\frac{s}{s_{\mathrm{o}}}\right)^{\alpha_{i}+\alpha_{j}-2},
$$

the polarisation may be rewritten as

$$
\left(\frac{d \sigma}{d t} P\right)_{\pi^{ \pm} \mathrm{p}}=(\sqrt{-t} / 16 \pi m)\left[X_{P P^{\prime}}+X_{P, A B C}+X_{P^{\prime}, A B C} \pm\left(X_{P \rho}+X_{P^{\prime} \rho}+X_{A B C, \rho}\right)\right] .
$$


from the experimental behaviour of the high-energy cross sections, our $\sigma_{i}(0)$ are positive except perhaps for the $A B C$ particle. Experiments give also ${ }^{8)} \alpha_{P}(0)=1, \alpha_{P^{\prime}}(0) \approx \alpha_{\omega}(0) \approx 0.4, \alpha_{p}(0) \approx 0.3$ and $\alpha_{A B C}(0) \leqslant 0$; further $\operatorname{Re}(\mathrm{d} \alpha / \mathrm{d} t) \approx m^{-2}$ for $t \geqslant 0$.

2. $\mathrm{Kp}$ and $\overline{\mathrm{K}} \mathrm{p}$ scattering. For elastic scattering, $\mathrm{K}^{ \pm}+\mathrm{p} \rightarrow \mathrm{K}^{ \pm}+\mathrm{p}$, one must put

$$
A \rightarrow A_{+}^{\mathrm{O}} \mp A_{-}^{\mathrm{O}} \pm A^{\prime}, \quad B \rightarrow B_{+}^{\mathrm{O}} \mp B_{-}^{\mathrm{O}} \pm B^{\prime},
$$

for charge exchange scattering, $\mathrm{K}^{-}+\mathrm{p} \rightarrow \overline{\mathrm{K}}^{\mathrm{O}}+\mathrm{n}$,

$$
A \rightarrow-2 A^{\prime}, \quad B--2 B^{\prime} \text {. }
$$

From isotopic spin, parity, and $G$ parity considerations, one has the trajectories $P, P^{\prime}$ and $A B C$ contributing to $A_{+}^{\mathrm{O}}(T=0, G=+$, parity $=+)$, $\omega$ to $A_{-}^{\mathrm{O}}(T=0, G=-$, parity $=-)$ and $\rho$ to $A^{\prime}(T=1, G=+$, parity $=-)$. Accordingly,

$$
\begin{aligned}
& A_{+}^{\mathrm{o}}=-\frac{s_{\mathrm{O}}}{2 m}\left\{\frac{1+\mathrm{e}^{-\mathrm{i} \pi \alpha_{P}}}{\sin \pi \alpha_{P}}\left(\frac{s}{s_{\mathrm{O}}}\right)^{\alpha P} c_{A P^{+}}+\frac{1+\mathrm{e}^{-\mathrm{i} \pi \alpha_{P^{\prime}}}}{\sin \pi \alpha_{P^{\prime}}}\left(\frac{s}{s_{\mathrm{O}}}\right)^{\alpha P^{\prime}} c_{A P^{\prime}}+\frac{1+\mathrm{e}^{-\mathrm{i} \pi \alpha_{A B C}}}{\sin \pi \alpha_{A B C}}\left(\frac{s}{s_{\mathrm{O}}}\right)^{\alpha_{A B C}} c_{A, A B C}\right\}, \\
& B_{+}^{0}=-\frac{1+\mathrm{e}^{-\mathrm{i} \pi \alpha_{P}}}{\sin \pi \alpha_{P}}\left(\frac{s}{s_{\mathrm{O}}}\right)^{\alpha_{P^{-1}}} c_{B P}-\frac{1+\mathrm{e}^{-\mathrm{i} \pi \alpha_{P^{\dagger}}}}{\sin \pi \alpha_{P^{\dagger}}}\left(\frac{s}{s_{\mathrm{O}}}\right)^{\alpha_{P^{t-1}}}-\frac{1+\mathrm{e}^{-\mathrm{i} \pi \alpha_{A B C}}}{\sin \pi \alpha_{A B C}}\left(\frac{s}{s_{\mathrm{O}}}\right)^{\alpha_{A B C^{-1}}} c_{B, A B C}, \\
& A_{-}^{\mathrm{o}}=\frac{s_{\mathrm{O}}}{2 m} \frac{1-\mathrm{e}^{-\mathrm{i} \pi \alpha_{\omega}}}{\sin \pi \alpha_{\omega}}\left(\frac{s}{s_{\mathrm{O}}}\right)^{\alpha_{\omega}} c_{A \omega}, \quad B_{-}^{\mathrm{o}}=\frac{1-\mathrm{e}^{-\mathrm{i} \pi \alpha_{\omega}}}{\sin \pi \alpha_{\omega}}\left(\frac{s}{s_{\mathrm{O}}}\right)^{\alpha_{\omega}-1} c_{B \omega}, \\
& A^{\prime}=\frac{s_{\mathrm{O}}}{2 m} \frac{1-\mathrm{e}^{-\mathrm{i} \pi \alpha_{\rho}}}{\sin \pi \alpha_{\rho}}\left(\frac{s}{s_{\mathrm{O}}}\right)^{\alpha_{\rho}} c_{A \rho}, \quad B^{\prime}=\frac{1-\mathrm{e}^{-\mathrm{i} \pi \alpha_{\rho}}}{\sin \pi \alpha_{\rho}}\left(\frac{s}{s_{\mathrm{O}}}\right)^{\alpha_{\rho}-1} c_{B \rho} .
\end{aligned}
$$

The polarisation, again depending only on interferences between different trajectories, behaves as

$$
P \sim S^{\alpha P^{t-\alpha_{P}}} \quad \text { or } \sim s^{\alpha_{\omega}-\alpha_{P}} \quad \text { or } \sim S^{\alpha_{\rho}-\alpha_{P}},
$$

depending on whether $P^{\dagger}, \omega$ or $p$ lie closest to $P$. Again $P_{\mathrm{K}^{+}}$and $P_{\mathrm{K}^{-} \mathrm{p}}$ have the same sign in the first case, opposite sign in the two latter cases, and there is no polarisation in charge exchange scattering. The total cross section is, with $c_{A i}+c_{B i}=\sigma_{i}^{K}(t)$

$\sigma_{\mathrm{K}^{ \pm} \mathrm{p}}^{\mathrm{tot}}=\sigma_{P}^{\mathbf{K}}(0)+\sigma_{P^{i}}^{\mathbf{K}}(0)\left(\frac{s}{s_{0}}\right)^{\alpha_{P^{\prime}}(0)-1}+\sigma_{A B C}^{\mathbf{K}}(0)\left(\frac{s}{s_{0}}\right)^{\alpha_{A B C}(0)-1} \mp \sigma_{\omega}^{\mathbf{K}}(0)\left(\frac{s}{s_{0}}\right)^{\alpha_{\omega}(0)-1} \pm \sigma_{\rho}^{\mathbf{K}}(0)\left(\frac{s}{s_{0}}\right)^{\alpha_{\rho}(0)-1}$

and the same remark applies as after eq. (12), except perhaps for the $A B C$ and $\rho$ particles.

I wish to thank Professor L. W. Jones and Professor M. L. Perl for discussions.

\section{References}

1) S.C. Frautschi, M.Gell-Mann and F.Zachariasen, Phys. Rev. 126 (1962) 2204.

See also: W. Kummer, CERN report 62-13 (1962), unpublished.

2) G. F. Chew, Revs. Modern Phys. 34 (1962) 394.

3) B. M. Udgaonkar, Phys. Rev. Letters 8 (1962) 142.

4) V.N.Gribov and I. Ya. Pomeranchuk, Phys. Rev. Letters 8 (1962) 412.
5) Y.Hara, Physics Letters 2 (1962) 246.

6) G. F. Chew et al., Phys. Rev. 106 (1957) 1337. See also: W.R. Frazer and J.R. Fulco, Phys. Rev. 117 (1960) 1603 for an extension to the crossed channel.

7) First ref. 1), appendix.

8) S.D.Drell, Rapporteur's talk to the 11th Int. HighEnergy Physics Conf. at CERN, July 1962 (unpublished). 